\title{
Intraoperative Hepatobiliary Injury
}

National Cancer Institute

\section{Source}

National Cancer Institute. Intraoperative Hepatobiliary Injury. NCI Thesaurus. Code C78382.

Damage to the hepatic parenchyma and biliary tract during a surgical procedure. 